\title{
Evaluation of Antivibration Glove Test Protocols Associated with the Revision of ISO 10819
}

\author{
Douglas D. REYNOLDS* and Eric WOLF
}

Center for Mechanical \& Environmental Systems Technology (CMEST), University of Nevada, Las Vegas, 4505

Maryland Parkway, Box 454027, Las Vegas, Nevada 89154, USA

Received January 31, 2005 and accepted June 14, 2005

\begin{abstract}
Effects of modifying the palm adapter geometry on ISO 10819 antivibration glove test results were investigated. 30 rigid acrylic adapters were designed, fabricated, and evaluated. The length of the adapters that met ISO 10819 requirements covered 70-80\% of the width of the palm. The upper curvatures of the adaptors were greater than or equal to the radius specified in ISO 10819:1996. Glove vibration transmissibility tests were conducted using a constant velocity vibration input with a value of $0.01 \mathrm{~m} / \mathrm{s}$ in each third octave frequency band from 16-1,600 $\mathrm{Hz}$ (F spectrum). Glove vibration transmissibility results obtained using the $M(16-400 \mathrm{~Hz})$ and $H(100-1,600 \mathrm{~Hz})$ spectra specified in ISO 10819:1996 were compared to similar results using the constant velocity spectrum. The results obtained from the constant velocity spectrum, when divided into the $M$ and $H$ frequency ranges, were nearly the same as those obtained from the ISO $10819 \mathrm{M}$ and $\mathrm{H}$ spectra. Test subject training was required to ensure reliable glove vibration transmissibility results. The measured transmissibility values obtained with the $M, H$, and $F$ spectra were all higher at the beginning of the test program. The transmissibility values decreased and approached lower limiting values as the test subjects became more experienced.
\end{abstract}

Key words: Antivibration gloves, ISO 10819, Palm adaptor, Hand adaptor, Glove vibration transmissibility

\section{Introduction}

Proposals for the future short-term and long-term revisions of ISO 10819 are being addressed by a joint ISO/CEN working group. Several issues are being addressed in this revision process. They include the adaptor design, the input vibration signal used to obtain the glove vibration transmissibility, the method in which the glove vibration transmissibility is obtained, and the effects of tests subjects on the measured glove vibration transmissibility. These issues were investigated and are reported in this paper.

Alternative palm adapter geometries, compared to the one defined in ISO 10819:1996, were investigated with the goal of addressing proper adapter alignment and fit in the palm of the hand during an ISO 10819 test. The initial design concept was that a smaller, better fitting palm adapter would

*To whom correspondence should be addressed. alleviate discomfort, facilitate proper gripping, be somewhat self-aligning in the palm, and would not affect the proper fit of the glove over the hand. Several different geometries were designed using Solid Works and produced using a Haas CNC milling machine at the University of Nevada, Las Vegas, Center for Mechanical and Environmental Technology. Adapters were designed with regards to critical dimensions and overall fit in the hand and then evaluated during glove vibration transmissibility tests.

A proposed change to the input vibration spectra in the forthcoming revision of ISO 10819 was investigated ${ }^{1}$. The current version of ISO 10819 uses two different spectra, named medium $(\mathrm{M})$ and high $(\mathrm{H})$ for their frequency content, as input signals. The proposed new method will be to use a constant $1 / 3$ octave band velocity input of $0.01 \mathrm{~m} / \mathrm{s}$ over the $1 / 3$ octave band frequency range of $16-1,600 \mathrm{~Hz}$. The use of a single input spectrum, rather than two separate spectra, will significantly reduce the testing and analysis 
Table 1. Palm adaptor design dimensions

\begin{tabular}{lccc}
\hline & \multicolumn{3}{c}{ Adapter dimensions (mm) } \\
& Min & Max & Special \\
\hline Length & 28 & 72 & \\
Bottom radius & 19 & 22 & 3-flats \\
Upper radius & 50 & 93 & flat \\
Thickness & 4.8 & 9.5 & \\
\hline
\end{tabular}

time necessary to evaluate the vibration transmissibility of antivibration gloves. The proper use of a single constantvelocity input spectrum hopefully will eliminate some of the disagreements that currently exist with the use of the $\mathrm{M}$ and $\mathrm{H}$ spectra specified in ISO 10819.

The final part of this paper presents a finding that became apparent as testing progressed. The effect of the subject familiarizing himself with proper testing technique is quantified and an explanation for this effect along with supporting data is reported.

\section{Materials and Methods}

\section{Palm adapter design}

Palm adapters were designed to increase test subject hand comfort when using the adapter for an ISO 10819 test. To accomplish this, palm adaptors were designed to more closely fit the contour of the test subject hand. Several combinations of dimensions were tried, including those that met the ISO 10819:1996 requirements. The critical dimensions that were adjusted to produce the different adapters were the overall adaptor length, the radius along the length of the upper adaptor profile, the bottom adaptor cross-sectional radius, and the adaptor thickness measured at the centerline of the adapter. The ranges of the dimensions that were investigated are given in Table 1. The special cases listed in Table 1 were also tried. These cases included completely flat upper profiles and flat bottom sections designed to maintain 2 lines of contact while keeping the accelerometer a short distance above the handle surface in combination with other standard dimensions. A few typical adapters that were made are shown in Fig. 1.

Glove vibration transmissibility test protocols and equipment

Figure 2 shows the test equipment used for the glove vibration transmissibility tests. The electro-dynamic shaker is a Tira Model TV 5550 LS. A Vibration View vibration controller was used to control the vibration signal to the
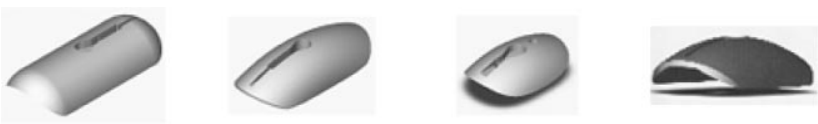

Fig. 1. Palm adaptor shapes.

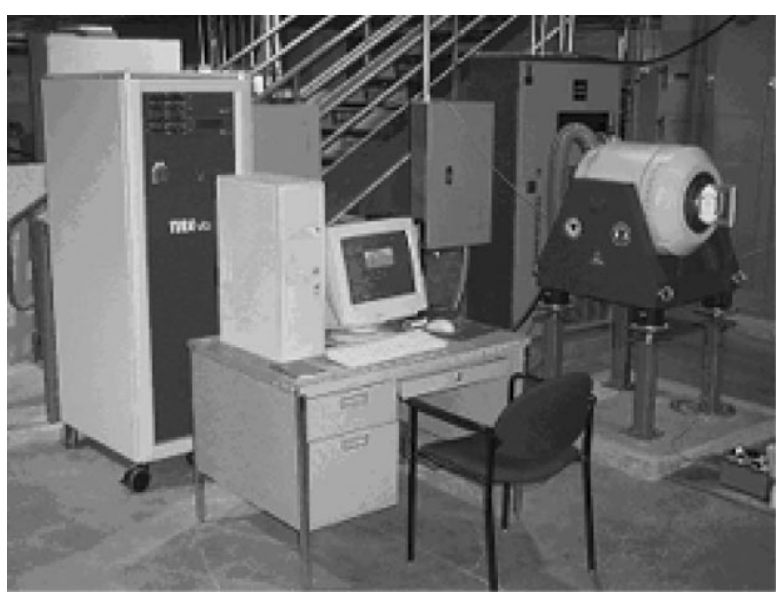

Fig. 2. CMEST vibration test setup.

electro-dynamic shaker. Monitoring, recording, and measuring the acceleration signals were performed with a Bruel and Kjaer Portable Pulse System and PCB 352C22 tear drop accelerometers. The grip force was monitored using handle-mounted strain gauge grip bar. The push force was monitored with a floor plate riding on bearings that was connected to a strain gauge proving-ring.

Three different test procedures were used in this project. The first was developed to quantify the performance of different adapter geometries with regards to linear transmissibility between the handle and palm adapter accelerometers. These tests were performed with the subject's bare hand applied to adapter/handle.

The second test procedure used was the ISO 10819 test protocol. Selected adapters were tested to determine differences in measurement of the mean transmissibility values of antivibration gloves.

The last test procedure was used to investigate a proposed change to the input vibration spectra of ISO 10819. The protocol is nearly identical to that of the standard, with the exception of a modified input spectrum and corresponding calculations.

\section{ISO 10819 test procedures}

ISO 10819 defines the test procedures for measuring the effectiveness of antivibration gloves. The requirements 


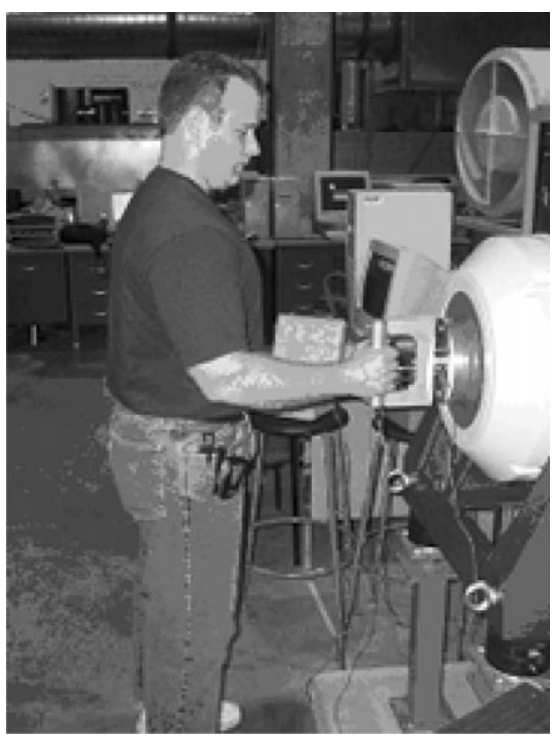

Fig. 3. Proper test posture.

specify proper test subject posture and preparation, the levels of push and grip force, the palm adapter geometry, minimum instrumentation requirements, and the methods for numerical analysis. ISO 0819 requires the use of three test subjects. They must be standing upright, dominant hand on the shaker handle with the palm adapter in between the handle and hand or inside the glove when one is tested. The angle of the elbow must be $90 \pm 10$ degrees. The wrist angle should be between 0-40 degrees. Proper test posture is illustrated in Fig. 3. During the $30 \mathrm{~s}$ test duration, the test subject is required to maintain $50 \pm 8 \mathrm{~N}$ of feed force on the handle and $30 \pm 5 \mathrm{~N}$ of grip force. Between each test, the subjects must break their grip on the handle.

The input acceleration spectra used for feedback control were the medium $(\mathrm{M})$ and high $(\mathrm{H})$ spectra specified in ISO 10819.

The calculations of mean corrected transmissibility values were obtained in the following manner. Two bare hand tests were conducted, and the results were linearly averaged for each adapter and spectra. The three test subjects then perform two tests each wearing an antivibration glove for each adapter and spectra. The rms. acceleration values for handle and palm adaptor accelerometer were measured for each test. The ISO-weighted acceleration values, $a_{w}$, for the M (16$400 \mathrm{~Hz})$ and $\mathrm{H}(100-1,600 \mathrm{~Hz})$ spectra were obtained by:

$$
a_{w}=\sqrt{a_{i} \times w_{i}}
$$

$\mathrm{a}_{\mathrm{i}}$ are the third octave band acceleration levels $\left(\mathrm{m} / \mathrm{s}^{2}\right)$ and $\mathrm{w}_{\mathrm{i}}$ are the ISO weighting values specified in ISO 53492). The ISO-weighted vibration transmissibility values for the bare

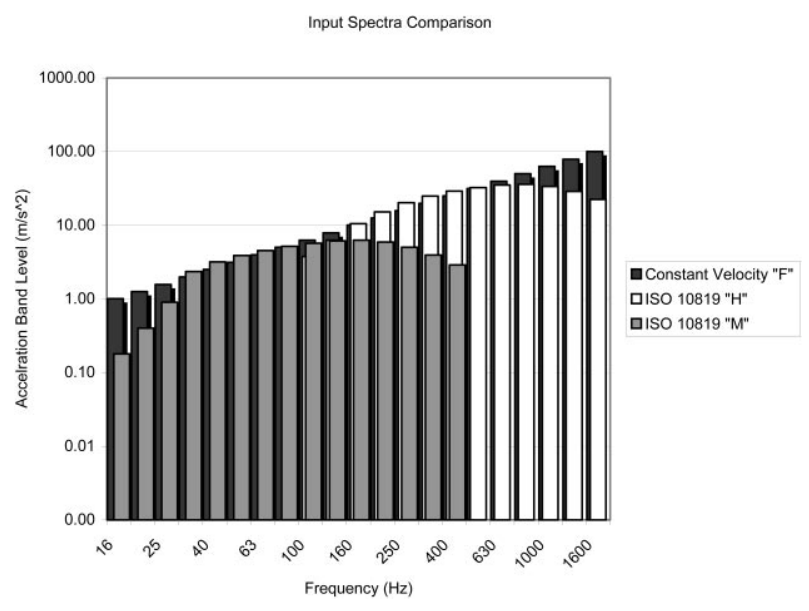

Fig. 4. F, H, and $M$ input spectra.

hand tests $\left(\operatorname{Tr}_{\text {iso(b) }}\right)$ and glove tests $\left(\mathrm{TR}_{\text {iso(g) }}\right)$ for each spectrum are:

$$
\begin{aligned}
& \operatorname{Tr}_{\text {iso(b) }}=\mathrm{a}_{\mathrm{w}(\mathrm{b} \text {-adaptor })} / \mathrm{a}_{\mathrm{w}(\mathrm{b} \text {-handle) }} \text { and } \\
& \operatorname{TR}_{\text {iso(g) }}=\mathrm{a}_{\mathrm{w}(\mathrm{g} \text {-adaptor })} / \mathrm{a}_{\mathrm{w}(\mathrm{g} \text {-handle })}
\end{aligned}
$$

$\mathrm{a}_{\mathrm{w} \text { (b-adaptor) }}$ is the ISO-weighted bare-hand adaptor acceleration, $\mathrm{a}_{\mathrm{w}(\mathrm{b}-\text {-handle) }}$ is the ISO-weighted bare hand handle acceleration, $a_{w(g-a d a p t o r)}$ is the ISO-weighted glove-hand adaptor acceleration, and $\mathrm{a}_{\mathrm{w}(\mathrm{g}-\mathrm{handle})}$ is the ISO-weighted glovehand handle acceleration. The ISO glove vibration transmissibility corrected for the bare hand vibration transmissibility is:

$$
\mathrm{TR}_{\text {iso }}=\mathrm{TR}_{\text {iso(g) }} / \mathrm{TR}_{\text {iso(b) }}
$$

The average ISO-weighted vibration transmissibility is the linear average of the six individual (two tests for each of three subjects) ISO-weighted vibration transmissibility values. For a glove to be an antivibration glove, the ISOweighted transmissibility must be less than 1.0 for the $\mathrm{M}$ spectrum and less than 0.6 for the $\mathrm{H}$ spectrum.

\section{Constant velocity spectrum test procedures}

A constant velocity input spectrum for glove vibration transmissibility tests is being proposed for the revision of ISO 10819. The input spectrum that is being proposed is a constant velocity $1 / 3$ octave band value of $0.01 \mathrm{~m} / \mathrm{s}$ in the $16-1,600 \mathrm{~Hz} 1 / 3$ octave frequency bands. This spectrum is referred to as spectrum $\mathrm{F}$ in this paper. Figure 4 shows graphs the F spectrum in relation to the ISO $10819 \mathrm{M}$ and $\mathrm{H}$ spectra.

The calculation of the vibration transmissibility of an antivibration glove is as follows:

Step 1: Measure the handle and palm adaptor $1 / 3$ octave band acceleration values for 12 tests: two bare hand and 
Table 2. Test subject characteristics for vibration transmissibility tests

\begin{tabular}{cccccc}
\hline Subject & Height & Weight & $\begin{array}{c}\text { Distance Base of } \\
\text { Palm to Finger Tip } \\
\mathrm{cm}\end{array}$ & $\begin{array}{c}\text { Palm } \\
\text { Width } \\
\mathrm{cm}\end{array}$ & $\begin{array}{c}\text { Hand } \\
\text { circum-ference } \\
\mathrm{cm}\end{array}$ \\
\hline EW & 1.95 & 107 & 20.3 & 10.2 & 23.5 \\
TW & 1.75 & 109 & 18.4 & 10.2 & 22.2 \\
CR & 1.65 & 70 & 17.8 & 9.5 & 21.6 \\
DR & 1.68 & 91 & 19.0 & 10.2 & 22.8 \\
\hline
\end{tabular}

two gloved hand tests for each of three test subjects.

Step 2: Divide the data into two groups. One set contains the $1 / 3$ octave band acceleration values from $16-400 \mathrm{~Hz}$ (FM spectrum) and the other contains the $1 / 3$ octave acceleration values from $100-1600 \mathrm{~Hz}$ (FH spectrum). The following steps were repeated for the FM and FH spectra separately.

Step 3: Calculate the ISO weighted glove vibration transmissibility values as described for equations (1) through (3).

\section{Palm adapter evaluation procedure}

Thirty different palm adapters were constructed and tested to explore the effects of adapter geometry on bare hand linear vibration transmissibility between the adaptor and the vibrating handle used for ISO 10819 tests. The input spectrum used for these tests was the F spectrum. Four test subjects performed two tests each. All other requirements and methods for performing the tests were the same as for performing the ISO 10819 bare hand tests. The criterion for adapter acceptability was for the linear transmissibility between the handle and adaptor acceleration signals in each $1 / 3$ octave frequency band between $16-1,600 \mathrm{~Hz}$, averaged over the 8 tests ( 4 subjects, tests each), to be between 0.95 and 1.05 .

\section{Linear vibration transmissibility tests}

The $0.01 \mathrm{~m} / \mathrm{s}$ constant velocity F spectrum input was used to measure the $1 / 3$ octave frequency band glove vibration transmissibility in the $1 / 3$ octave band frequency range of 16-1,600 Hz. The bare hand palm adaptor vibration transmissibility was measured. The gloved-hand vibration transmissibility was then measured. The measured glove vibration transmissibility was corrected for the bare hand adaptor vibration transmissibility as specified in ISO 10819 . Two bare hand adaptor transmissibility tests were conduct for each of three test subjects. Two gloved hand vibration transmissibility tests were conducted for each of three test subject.

\section{Discussion of Results}

The adapters were evaluated in two phases. Phase 1 determined whether or not the palm adapters met the acceptability criterion. Phase 2 investigated the effect of the adapter shape on the glove vibration transmissibility values, calculated for the ISO $10819 \mathrm{M}$ and $\mathrm{H}$ spectra.

\section{Bare hand palm adaptor vibration transmissibility}

The results of the bare hand linear vibration transmissibility tests were categorized into two groups. The graphs depict the $1 / 3$ octave band linear transmissibility values averaged over 8 tests ( 4 subjects, 2 tests each). The four test subjects had the characteristics shown in Table 2. The error bars in the plots are \pm one standard deviation.

The first group of adaptors met the acceptability criteria, which were linear vibration transmissibility values between the adaptor and handle in each $1 / 3$ octave band (averaged over 4 test subjects) of between 0.95 and 1.05. Seven of the thirty adaptors that were examined met the acceptability criteria. Figure 5 shows a representative vibration transmissibility plot of these adapters.

Palm adaptors that met the acceptability criteria had some common characteristics. Their overall length covered 70$80 \%$ of the width of the palm. The radius of the upper adaptor curvature was greater than or equal to the upper adaptor curvature radius specified in ISO 10819:1996. The radius of curvature of the bottom side of the adaptor ranged from 19-22 mm.

Figures 6 through 8 show typical vibration transmissibility plots of palm adaptors that did not meet the acceptability criteria.

\section{ISO 10819 glove vibration transmissibility tests}

The second series of tests were glove vibration transmissibility tests. Tests were conducted per the procedures specified in ISO 10819. Tests were then conducted using a $0.01 \mathrm{~m} / \mathrm{s}$ constant velocity vibration input. Appropriate ISO 10819 test procedures were used with the 
Adaptor 2

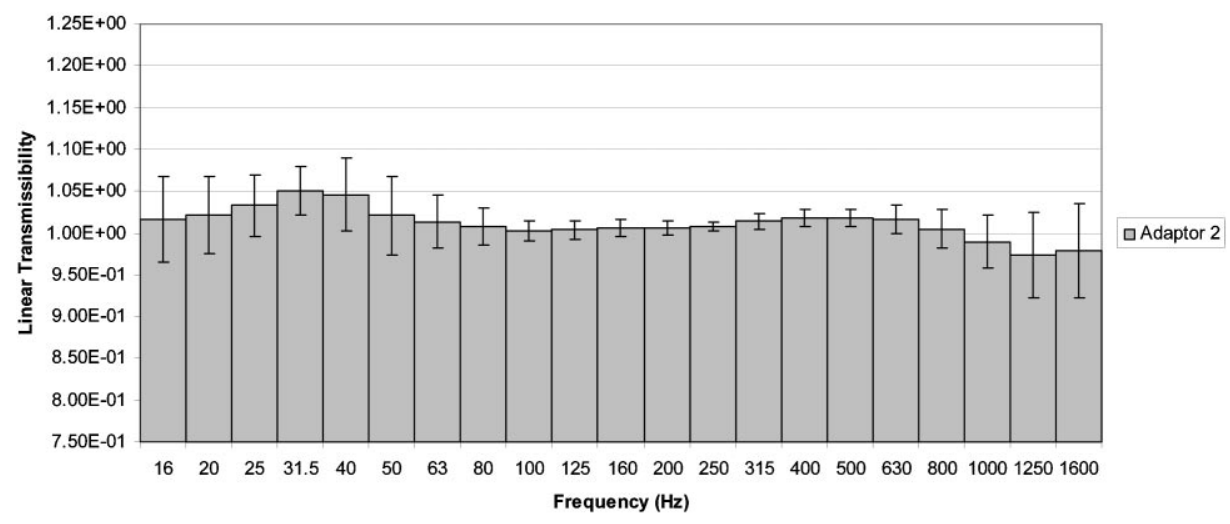

Fig. 5. Adaptors that met acceptability Criteria.

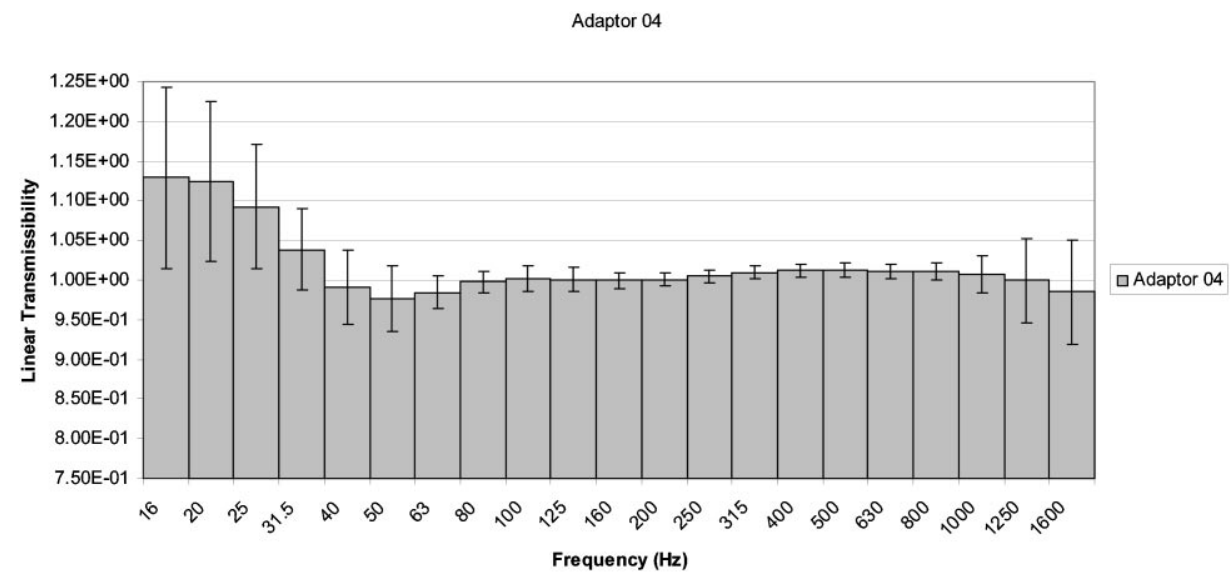

Fig. 6. Adaptors with low frequency deficiencies.

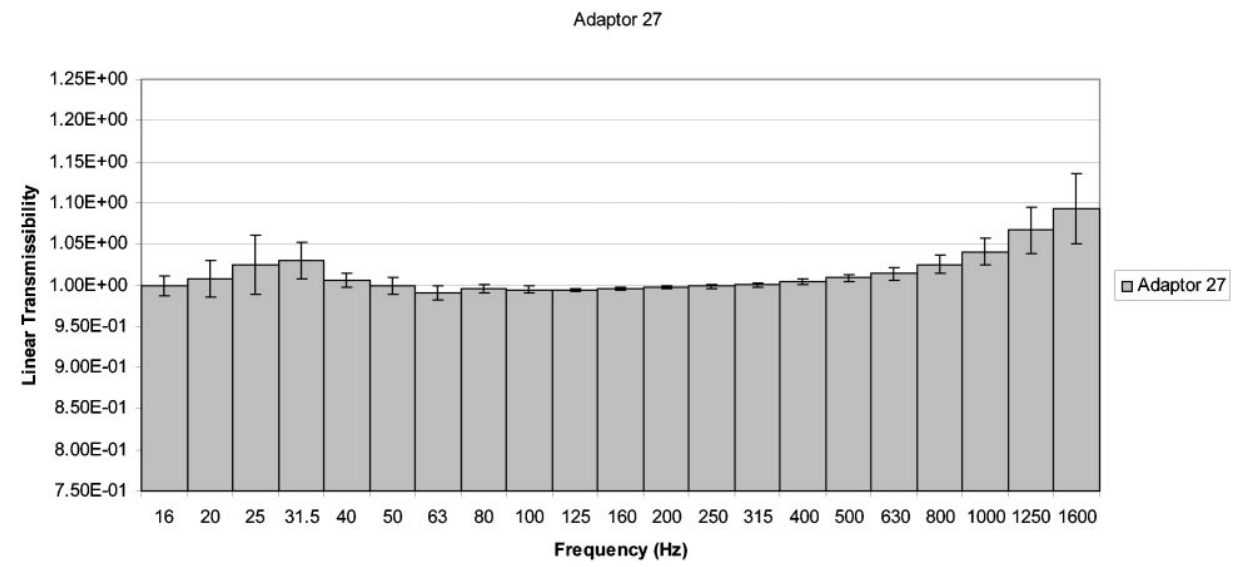

Fig. 7. Adaptors with high frequency deficiencies. 


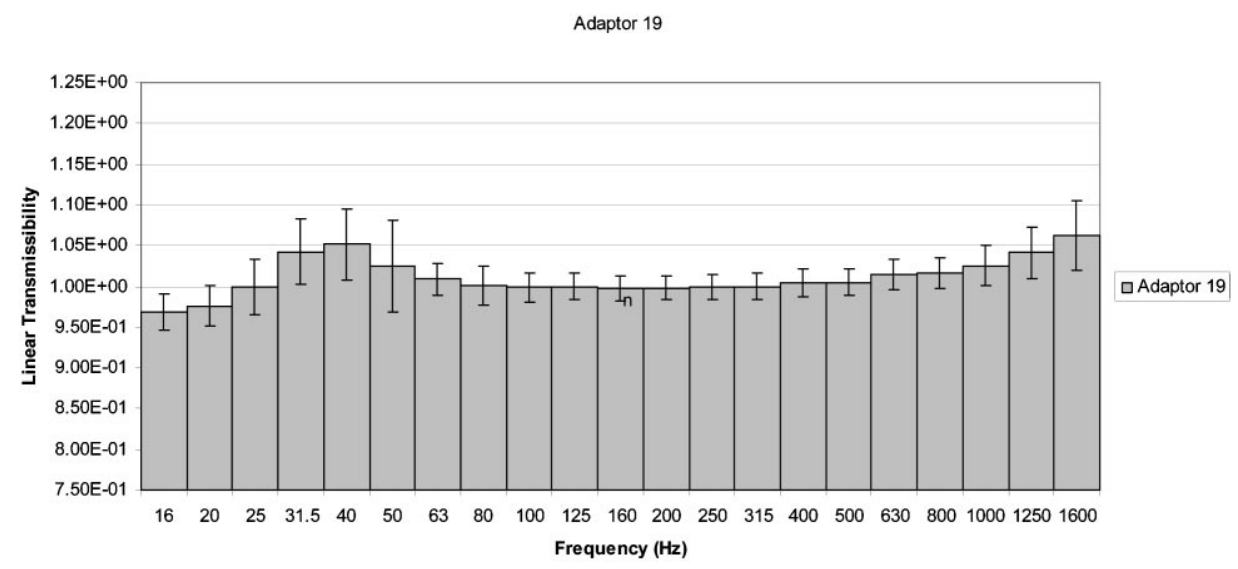

Fig. 8. Adaptors with mid and high frequency deficiencies.

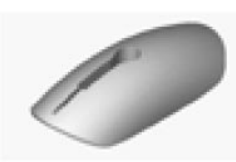

A02

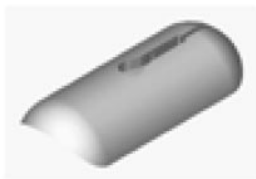

A03

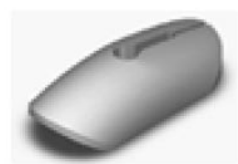

A29

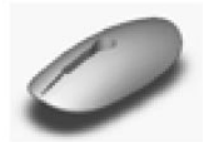

A30

Fig. 9. Palm adaptors - met acceptability Criteria.

Table 3. Glove vibration transmissibility (Adaptors met acceptability criterion)

\begin{tabular}{lccccccc} 
H spectrum & \multicolumn{7}{c}{ FH spectrum } \\
\hline & Glove 1 & Glove 2 & Glove 3 & & Glove 1 & Glove 2 & Glove 3 \\
\hline A02 & 0.80 & 0.73 & 0.59 & A02 & 0.75 & 0.64 & 0.53 \\
A03 & 0.81 & 0.67 & 0.51 & A03 & 0.72 & 0.63 & 0.49 \\
A29 & 0.83 & 0.67 & 0.51 & A29 & 0.73 & 0.60 & 0.49 \\
A30 & 0.80 & 0.70 & 0.53 & A30 & 0.75 & 0.64 & 0.50 \\
\hline
\end{tabular}

M spectrum $\quad$ FM spectrum

\begin{tabular}{cccccccc}
\hline & Glove 1 & Glove 2 & Glove 3 & & Glove 1 & Glove 2 & Glove 3 \\
\hline A02 & 0.86 & 0.80 & 0.64 & A02 & 0.87 & 0.82 & 0.73 \\
A03 & 0.87 & 0.79 & 0.63 & A03 & 0.85 & 0.80 & 0.70 \\
A29 & 0.86 & 0.80 & 0.66 & A29 & 0.87 & 0.79 & 0.71 \\
A30 & 0.87 & 0.79 & 0.64 & A30 & 0.88 & 0.81 & 0.71 \\
\hline
\end{tabular}

constant velocity vibration input. Three commercially available antivibration gloves were used in this project. Glove 1 used a gel foam elastomeric element, glove 2 used a $\mathrm{Nu}_{2} \mathrm{O}_{2}$ polymer elastomeric element, and glove 3 used an air-bladder elastomeric element. Four palm adaptors that met the acceptability criteria were used for these tests. They were designated adaptors A02, A03, A29, and A30 (Fig. 9). The characteristics of the three test subjects that were used for these tests are shown in Table 2. The test subjects were
TW, CR, and DR.

Table 3 shows a comparison of the glove vibration transmissibility values calculated for each adapter/glove combination. The $\mathrm{M}$ and $\mathrm{H}$ spectra results were obtained using the test procedures specified in ISO 10819. The FM and FH spectra results were obtained using the $0.01 \mathrm{~m} / \mathrm{s}$ constant velocity input and appropriate ISO 10819 test procedures. The results show that for all gloves and input spectra, the related glove vibration transmissibility values 


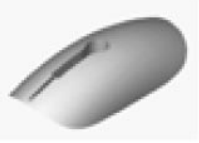

A19

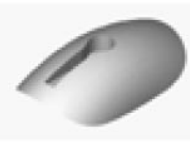

A20
Fig. 10. Palm adaptors - did not meet acceptability criteria.

do not significantly vary among the adaptors that were tested. The largest variation is found in the $\mathrm{H}$ spectra for glove 3 , which ranges from 0.59 to 0.51 . A range of 0.08 in this type of measurement is somewhat significant, but it is isolated to one adapter in one particular spectrum.

No single adapter consistently yielded the highest or lowest glove vibration transmissibility values for all spectra. The small variations of the glove vibration transmissibility values were well distributed among the adapters. The data suggest that, for adaptors that meet the adaptor acceptability criterion, there will be very small variations in the glove vibration transmissibility values obtained with different adaptors.

Two adapters that did not meet the acceptability criterion were also tested. They are designated adaptors A19 and A20 (Fig. 10). Adapter 19 failed in the $40 \mathrm{~Hz} \mathrm{1/3}$ octave frequency band and in the $1,600 \mathrm{~Hz}$ third octave band.
Adapter 20 failed in the 1,250 and $1,600 \mathrm{~Hz} 1 / 3$ octave frequency bands. The glove vibration transmissibility values are reported in Table 4.

The calculated glove vibration transmissibility values for adaptors A19 and A20 lie within the respective ranges of the other four adaptors for all input spectra. The data confirm that even though an adaptor may slightly amplify the bare hand acceleration signal, the effect is negligible when the glove transmissibility values are properly corrected for the bare hand adaptor vibration transmissibility. The ISO 5349 weighting values increasingly weights the higher frequency $1 / 3$ octave frequency band transmissibility values out. Thus their effect is greatly diminished. More testing is required to fully define the limit of bare hand adaptor transmissibility amplification that will not significantly affect glove vibration transmissibility values.

A comparison can be made between the $\mathrm{H}$ and $\mathrm{FH}$ spectra data and the $\mathrm{M}$ and FM spectra data. Table 5 shows this comparison. When comparing the $\mathrm{H}$ and $\mathrm{FH}$ data, the glove vibration transmissibility values obtained using the $\mathrm{FH}$ input spectra tend to yield slightly lower glove vibration transmissibility values. The range of these differences varies between the gloves that were tested. When comparing the FM and M spectra, the ranges of values between the two

Table 4. Glove vibration transmissibility (Adaptors failed acceptability criterion)

H spectra

FH spectra

\begin{tabular}{cccccccc}
\hline & Glove 1 & Glove 2 & Glove 3 & & Glove 1 & Glove 2 & Glove 3 \\
\hline A19 & 0.83 & 0.70 & 0.55 & A19 & 0.75 & 0.66 & 0.51 \\
A20 & 0.81 & 0.67 & 0.54 & A20 & 0.75 & 0.61 & 0.52 \\
\hline
\end{tabular}

M spectra $\quad$ FM spectra

\begin{tabular}{cccccccc}
\hline & Glove 1 & Glove 2 & Glove 3 & & Glove 1 & Glove 2 & Glove 3 \\
\hline A19 & 0.86 & 0.79 & 0.62 & A19 & 0.88 & 0.83 & 0.71 \\
A20 & 0.84 & 0.76 & 0.66 & A20 & 0.86 & 0.78 & 0.72 \\
\hline
\end{tabular}

Table 5. Glove vibration transmissibility values (Six-adapter average)

\begin{tabular}{lccccccc}
\multicolumn{7}{c}{ H spectra } & \multicolumn{7}{c}{ FH spectra } \\
\hline & Glove 1 & Glove 2 & Glove 3 & & Glove 1 & Glove 2 & Glove 3 \\
\hline AVG & 0.81 & 0.69 & 0.54 & AVG & 0.74 & 0.63 & 0.51 \\
\hline \multicolumn{7}{l}{ M spectra } & \multicolumn{7}{l}{ FM spectra } \\
\hline \multicolumn{2}{l}{ Glove 1 } & Glove 2 & Glove 3 & & Glove 1 & Glove 2 & Glove 3 \\
\hline AVG & 0.86 & 0.79 & 0.64 & AVG & 0.87 & 0.80 & 0.71 \\
\hline
\end{tabular}


spectra are much smaller on average than was the case with $\mathrm{FH}$ and $\mathrm{H}$ spectra. Given the expected variability between glove vibration transmissibility tests, the results are essentially the same for the FM and M spectra.

\section{Linear glove vibration transmissibility tests}

Figures 11 through 13 show the linear glove vibration transmissibility results for gloves 1 through 3 , respectively. The figures show the results that were obtained for the six palm adaptors that were examined. Four of the adaptors met the adaptor acceptability criterion. Two failed the criterion. The transmissibility values for each adaptor are the averages of six individual tests (two tests from each of three test subjects). The average values of linear vibration transmissibility in the low frequency $1 / 3$ octave frequency bands (16-40 Hz) showed a slight amplification for some gloves/adapter combination.

\section{Test subject training}

In the course of performing the vibration transmissibility tests reported in this paper, a general numerical trend was found to exist for each subject from test to test. All measurement equipment, calibrations, adapters, and gloves were the same for each series of tests. To ensure measurement consistency in the vibration transmissibility tests, all tests were repeated several times over a period of days and weeks. With each round of repetitions, the glove vibration transmissibility values for all individual test subjects continually decreased until limiting values were approached. These limiting values were close to corresponding values that were measured for each glove by other laboratories. This effect was not immediately present, but appeared when test data from previous days were compared to newer test data. Regardless of how many times a test was repeated over the course of a single day, the test values did not show appreciable variation. However, differences appeared on a day-to-day or week-to-week basis. This is attributed to a process of "familiarization" of the test subjects to the correct feel of the adapter inside the glove. There was little variation in the bare hand tests, because it is easier to align the adapter the same way every time when it can be seen. Table 6 is a compilation of glove vibration transmissibility values for each glove and spectra over two test runs. The effect is less pronounced in the $\mathrm{H}$ spectra data because those tests were run at the end of round 1. Only adapters 02 and 29 are presented.

By examining these results, it becomes clear that each subject became more experienced in proper glove testing methods as testing progressed over a period of several weeks.

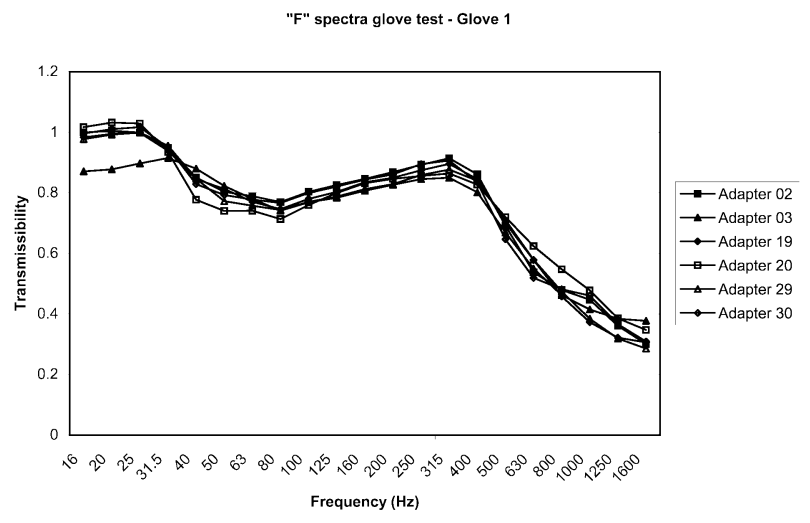

Fig. 11. Linear vibration transmissibility, glove 1 .

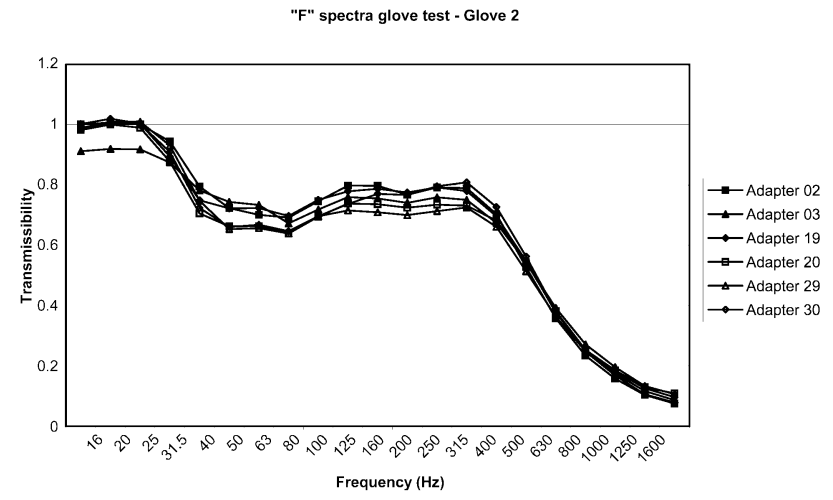

Fig. 12. Linear vibration transmissibility, glove 2 .

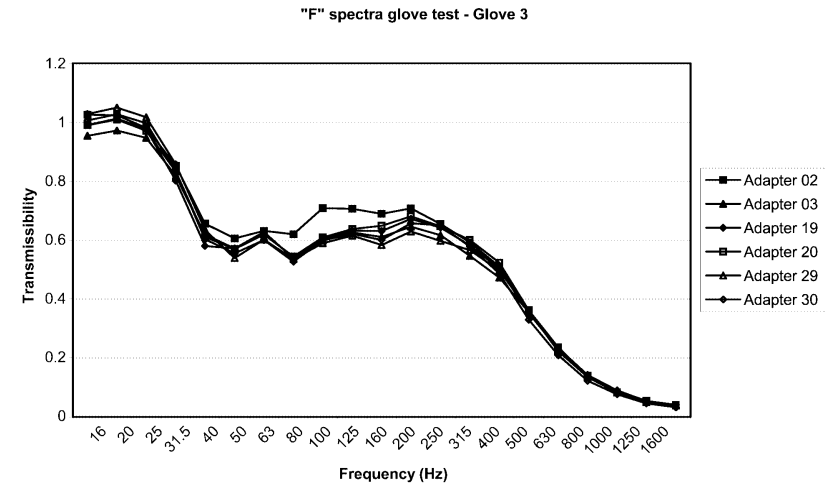

Fig. 13. Linear vibration transmissibility, glove 3 .

As a consequence, their ability to correctly perform the tests increased. This effect was present for all test subjects. These results suggest that it would be improper to use a test subject for antivibration glove testing without proper training and exposure to the appropriate test procedures.

It is necessary to "calibrate" the test subjects to obtain correct glove vibration transmissibility results. The easiest 
Table 6. Test subject training comparison

Round 1

F Spectra

\begin{tabular}{cccccccc}
\hline & glove 1 & glove2 & glove3 & & glove 1 & glove2 & glove3 \\
\hline A02 & 0.77 & 0.73 & 0.72 & A02 & 0.81 & 0.74 & 0.65 \\
A29 & 0.77 & 0.70 & 0.71 & A29 & 0.80 & 0.71 & 0.64 \\
\hline
\end{tabular}

\begin{tabular}{lccccccc}
\multicolumn{7}{c}{ FM Spectra } & \multicolumn{7}{c}{ FM Spectra } \\
\hline & glove 1 & glove2 & glove3 & & glove 1 & glove2 & glove3 \\
\hline A02 & 0.85 & 0.83 & 0.81 & A02 & 0.87 & 0.82 & 0.73 \\
A29 & 0.87 & 0.81 & 0.81 & A29 & 0.87 & 0.79 & 0.71 \\
\hline
\end{tabular}

$\begin{array}{ll}\text { FH Spectra } & \text { FH Spectra }\end{array}$

\begin{tabular}{cccccccc}
\hline & glove 1 & glove2 & glove3 & & glove 1 & glove2 & glove3 \\
\hline A02 & 0.70 & 0.65 & 0.64 & A02 & 0.75 & 0.64 & 0.53 \\
A29 & 0.69 & 0.60 & 0.61 & A29 & 0.73 & 0.60 & 0.49 \\
\hline
\end{tabular}

\begin{tabular}{lccccccr}
\multicolumn{1}{c}{ H Spectra } & \multicolumn{7}{c}{ H Spectra } \\
\hline & glove 1 & glove2 & glove3 & & glove 1 & glove2 & glove3 \\
\hline A02 & 0.88 & 0.80 & 0.72 & A02 & 0.80 & 0.73 & 0.59 \\
A29 & 0.84 & 0.77 & 0.71 & A29 & 0.83 & 0.67 & 0.51 \\
\hline
\end{tabular}

\begin{tabular}{cccccccc}
\multicolumn{1}{c}{ M Spectra } & \multicolumn{7}{c}{ M Spectra } \\
\hline & glove 1 & glove2 & glove3 & & glove 1 & glove2 & glove3 \\
\hline A02 & 0.83 & 0.71 & 0.65 & A02 & 0.86 & 0.80 & 0.64 \\
A29 & 0.79 & 0.68 & 0.63 & A29 & 0.86 & 0.80 & 0.66 \\
\hline
\end{tabular}

method to gauge proper subject performance is to test a glove with reasonably well-known glove vibration transmissibility values. Considering the high degree of variability introduced by the test subject's physical nature, obtaining a clear picture of a correct vibration transmissibility value for a specific test subject and glove may not be easy. It is necessary to look at average values, and then determine a proper confidence interval. Further study on this matter is needed to define a complete procedure for properly training a test subject.

\section{Conclusions}

1. Adapter geometry can significantly affect the outcomes of bare hand palm adaptor linear vibration transmissibility. The performance of an adapter is heavily influenced by the curvature of the upper profile and the fit of the adapter to the handle. A curved bottom surface that matches the diameter of the handle is recommended. An adapter's length must span at least $70-80 \%$ of the width of the palm.

2. The geometry of the palm adapter does not affect the glove vibration transmissibility value for an antivibration glove if the average linear transmissibility value for the adaptor falls within the range of 0.95 to 1.05 in each $1 /$ 3 octave frequency band from $16-1,600 \mathrm{~Hz}$. Several adapter geometries that were tested met this requirement, including the ISO 10819 defined adapter.

3. The adaptors that do not meet the linear vibration transmissibility criterion will give accurate glove vibration transmissibility values when the glove vibration transmissibility test results are adjusted for the bare hand adaptor vibration transmissibility.

4. It is possible to replace the $\mathrm{M}$ and $\mathrm{H}$ spectra currently defined as inputs in ISO 10819 with a single F spectra that consists of a $0.01 \mathrm{~m} / \mathrm{s}$ constant velocity in each of 
the $1 / 3$ octave frequency bands between $16-1,600 \mathrm{~Hz}$. The glove vibration transmissibility values obtained using the F spectra were nearly identical to the those obtained using the ISO $10819 \mathrm{M}$ and $\mathrm{H}$ spectra when the F spectra was mathematically divided into the $\mathrm{M}$ and $\mathrm{H}$ spectra frequency ranges.

5. Test subjects must be trained in order to achieve acceptable repeatable glove vibration transmissibility test results. It is important for the test subject to perform many tests to become accustomed to the feel of the palm adapter in a glove.

\section{References}

1) International Organization for Standardization (1996) Mechanical vibration and shock-Hand-arm vibration-Method for the measurement and evaluation of the vibration transmissibility of gloves at the palm of the hand. ISO 10819:1996.

2) International Organization for Standardization (2001) ISO 5349-1. Mechanical vibration and shockMeasurement and evaluation of human exposure to hand-transmitted vibration-Part 1: General requirements. 